\section{More likely than not}

\author{
Stephen B Hanauer
}

I recently had the opportunity to testify in the US Federal Court regarding the potential for the measles-mumps-rubella (MMR) vaccine to cause Crohn's disease. The testimony was a component of the larger claim that the MMR vaccine causes autism, and there is a spinoff claim by some pediatricians that autism is associated with a unique form of intestinal inflammation, so-called autistic enterocolitis.

I was, at first, curious as to why (and how) the legal system was the entity to determine causation, rather than a consensus amongst scientists. Indeed, reports from the Immunization Safety Review Committee, which were requisitioned by the Institute of Medicine in 2001 and 2004, reviewed data from published and unpublished epidemiological studies regarding causality and the potential mechanisms by which immunization might cause autism (and Crohn's disease). These reports concluded that the body of epidemiological evidence favored rejection of a causal relationship for both the MMR vaccine and the preservative thiomersal (known in the US as thimerosal). Furthermore, recent reports of a potential association between the MMR vaccine, gastrointestinal inflammation and developmental regression have created even more furor in the media, more so in the UK than in the US. These claims have also been refuted by critical scientific analyses (Histopathology [2007] 50: 371-379 and Cochrane Database of Systematic Reviews 2005, Issue 4. Art. No.: CD004407. DOI: 10.1002/14651858.CD004407.pub2).

The claim for an association between the MMR vaccine and autism is being adjudicated in the Federal Court based on US law that provides compensation for vaccine-related injuries. We recall the differences in outcomes from the two OJ Simpson trials-one outcome

\section{...neither \\ atypical \\ mycobacteria \\ nor MMR \\ vaccines meet \\ the scientific \\ criteria for \\ causation - \\ independent \\ of the legal \\ conclusions}

SB Hanauer is Editorin-Chief of Nature

Clinical Practice

Gastroenterology \&

Hepatology.

\section{Competing interests}

The author declared no

competing interests.

www.nature.com/clinicalpractice doi:10.1038/ncpgasthep0923 was based on "reasonable doubt" and the other on the "preponderance of the evidence". The evidence in the US courts requires a judgment based on "more likely than not" or a greater than $50 \%$ chance that the vaccine caused the adverse outcomes.

The testimony I gave involved the case of a young girl who developed symptoms of autism shortly after receiving the MMR vaccine. She subsequently developed a spectrum of gastrointestinal symptoms (GERD, constipation, diarrhea) and findings (lymphoid hyperplasia and ileocolonic aphthae) associated with extraintestinal inflammatory disorders (uveitis and arthritis). I did not agree that there was sufficient evidence for a diagnosis of Crohn's disease and, similarly, feel that the evidence for a unique form of autistic enterocolitis is also weak. The findings of ileocolonic aphthae could have been attributed to the concurrent use of NSAIDs, which is a troublesome differential diagnosis seen in many patients who have diarrheal symptoms and wireless capsule endoscopy findings of small bowel aphthae.

Over the past several decades claims that Crohn's disease is caused by atypical mycobacteria have led to fears in the media and the public regarding the safety of the milk supply and, most recently, the claim regarding the risks of MMR vaccination has led to concerns regarding vaccinations. To support these associations, Helicobacter pylori is frequently given as an example of paradigm shifts in attributable causation. Fortunately, the $H$. pylori hypothesis was accepted only after confirmation of Koch's postulates. To date, neither atypical mycobacteria nor MMR vaccines meet the scientific criteria for causation-independent of the legal conclusions. 